\title{
Analysis of Automated Contrast Checking Tools
}

\author{
Rafael Almeida \\ rafael76almeida@gmail.com \\ Faculdade de Ciências, Universidade de Lisboa \\ Lisboa, Portugal
}

\begin{abstract}
Creating web pages with information that is perceivable has to be a priority concern in their development. One of the main factors impacting the ability to read text in web pages is the contrast between the text's foreground and background colors. A number of automated tools have emerged to help web page designers create pages that abide by the minimum contrast requirements, established in the Web Content Accessibility Guidelines. We studied a subset of these tools to understand their current limitations. To help us achieve this goal we created a set of test cases exploring the different ways in which foreground and background colors can be mixed in web pages. We found that current tools can assist in detecting poor contrast in web pages, but have a clear limitation when evaluating text over images and text inside images.
\end{abstract}

\section{CCS CONCEPTS}

- Human-centered computing $\rightarrow$ Accessibility design and evaluation methods; Accessibility systems and tools.

\section{KEYWORDS}

color contrast, text, accessibility, automated evaluation

\section{ACM Reference Format:}

Rafael Almeida and Carlos Duarte. 2020. Analysis of Automated Contrast Checking Tools. In 17th Web for All Conference (W4A '20), April 20-21, 2020, Taipei, Taiwan. ACM, New York, NY, USA, 4 pages. https://doi.org/10.1145/ 3371300.3383348

\section{INTRODUCTION}

Insufficient contrast between text color and background color is a widespread problem throughout the web [4, 7]. The ability to quickly navigate and perceive information on a web page can be severely constrained by the lack of compliance with the recommendations set out in the Web Content Accessibility Guidelines ${ }^{1}$ (WCAG) success criterion 1.4.3 Contrast (Minimum) and success criterion 1.4.6 Contrast (Enhanced).

While the WCAG aim to ensure equal access to information for all users, success criteria 1.4.3 and 1.4.6 are directed at people with low vision who often have difficulty reading text that does not contrast with its background. This problem can be exacerbated if

\footnotetext{
$\overline{{ }^{1} \text { https://www.w3.org/TR/WCAG21/ }}$

Permission to make digital or hard copies of part or all of this work for personal or classroom use is granted without fee provided that copies are not made or distributed for profit or commercial advantage and that copies bear this notice and the full citation on the first page. Copyrights for third-party components of this work must be honored

For all other uses, contact the owner/author(s)

W4A '20, April 20-21, 2020, Taipei, Taiwan

(C) 2020 Copyright held by the owner/author(s)

ACM ISBN 978-1-4503-7056-1/20/04.

https://doi.org/10.1145/3371300.3383348
}

\author{
Carlos Duarte \\ caduarte@campus.ul.pt \\ LASIGE, Faculdade de Ciências, Universidade de Lisboa \\ Lisboa, Portugal
}

the person has a color vision deficiency that lowers the contrast even further. Vision deficiency can result from traumatic events or birth conditions, but it is also one of the consequences of ageing that leads to decreased visual ability in general and in the color palette that can be detected $[2,8]$. With the increasingly ageing population, the number of web users that are impacted by this problem is only likely to increase. However, ensuring that minimum contrast ratios are respected in published content benefits not only those users, but all users, irrespective of their visual abilities, who read text on small, dimmer screens, like those in the smartphones used by a large percentage of the population of web users.

To help designers create content that meet the established guidelines, several tools have been developed to automate color contrast checking. In this paper, we report the results of a study of a subset of those tools. In the following section, we briefly review previous studies of color contrast issues. This is followed by a description of the tools that have been studied. A description of the test cases created for this study precedes the presentation of the results. The paper concludes with a discussion of the findings.

\section{RELATED WORK}

The growing concern about accessibility has led to numerous studies on the contrast between text and background color on web pages. Those studies covered different topics: how color contrast affects information gathering, the impact of contrast on users with color blindness, tools to correct detected contrast problems or even tools to suggest colors that allow for positive contrast.

Tools have been developed and studied that, after picking a color for the foreground or background, are capable of suggesting a color combination between foreground and background color that has acceptable contrast according to the WCAG contrast recommendations [3,6]. A study with people with color blindness has been done to understand the difficulties and barriers that exist in the use of web pages by these individuals and what recommendations to consider when developing web pages [1]. The impact of contrast between text and background colors on digital text reading has been studied in [9]. A way to compensate for contrast issues and optimize the contrast of a web page has been studied in [5].

A study comparing the various tools and trying to understand their limitations still does not exist, to the best of our knowledge. We try to address that limitation in this paper.

\section{CONTRAST CHECKING TOOLS}

The Web Accessibility Initiative (WAI) provides a list ${ }^{2}$ of tools that are capable of automatically checking for multiple accessibility problems. Some of those tools can check for color contrast issues. However, most of the tools available require their users to enter

$\overline{{ }^{2} \text { https://www.w3.org/WAI/ER/tools/ }}$ 


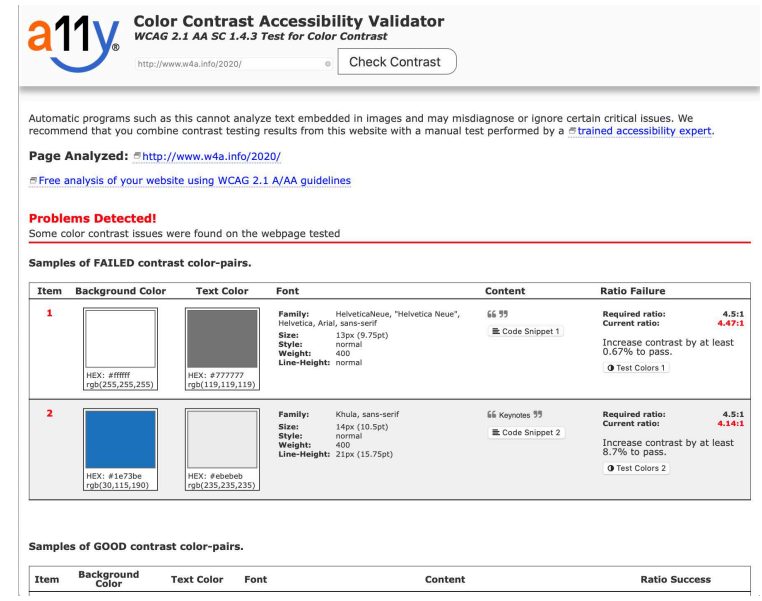

Figure 1: Screenshot of the evaluation of the W4A home page with the Color Contrast Accessibility Validator.

a foreground color and a background color and then proceed to calculate their contrast, indicating whether it respects the WCAG recommendation or not. Given the possibly large number of colors that can be in use in a single web page, requiring the page designer to test the different color combinations can be taxing. Additionally, for entities that need to check contrast issues on monitored web pages, this approach requires the human evaluator to find all color combinations and check each one individually, which is also a burdensome task. For this reason, in this study we focused on the subset of tools that can access a web page and automatically detect contrast issues within that web page. All the tools considered are free. In the following paragraphs we introduce the tools we found that meet this criteria.

\subsection{Color Contrast Accessibility Validator}

The Color Contrast Accessibility Validator ${ }^{3}$ (figure 1), from the Bureau of Internet Accessibility, Inc., is a website that receives a URL and generates a report with the results of the tests performed on the page. The results generated are divided into two categories, the first containing the detected problems and the second containing the cases where the detected contrast is according to the standards. Tests are cataloged with a number for each of the categories (success / failure), marking the detected background and foreground colors. The font, size, style, and font weight of the text being tested is also indicated. Finally, the ratio obtained and the ratio required (to be compliant) for that type of text is indicated. This tool also has a color pair test on its homepage, that allows the user to choose a background color and a foreground color to test their contrast ratio.

\subsection{WAVE}

The WAVE Web Accessibility Tool ${ }^{4}$ (figure 2), from WebAIM, is also a website that receives a URL that is then used to make a request to the page to be tested before generating a report with the accessibility evaluation. This report renders the tested web page

\footnotetext{
${ }^{3} \mathrm{https} / / /$ color.a11y.com

${ }^{4}$ https://wave.webaim.org
}

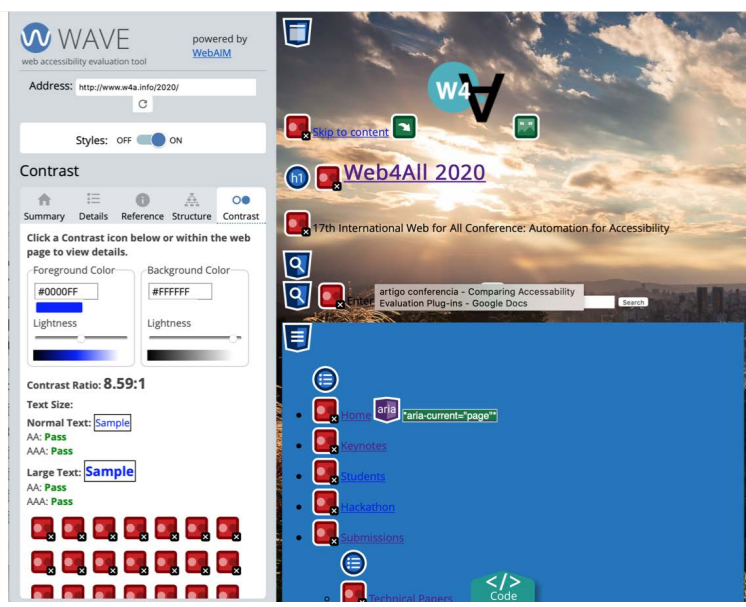

Figure 2: Screenshot of the evaluation of the W4A home page with WAVE.

with the evaluation results overlaid on the elements, that is, with icons above the tested elements representing the evaluation result. By clicking on the icons we can see a detailed report on the left side of the page showing the contrast value, the colors used and even text samples in different sizes. The tool also allows access to the code of the page being tested, and by clicking on an error icon it highlights to which part of code the element being tested refers to. This tool also allows us to test without any style being assigned to the elements, simulating what the page would be like if the styles could not be rendered.

\subsection{WCAG Color Contrast Checker}

The WCAG Color Contrast Checker (figure 3) is a browser extension (available for Google Chrome ${ }^{5}$ and Mozilla Firefox ${ }^{6}$ ) that can dynamically access the page (with any changes to be immediately recognized) and produce a report of tests made on the page. Results include the elements (including hidden elements) that were tested, their background and foreground colors, and the value of the contrast obtained. The elements that have the same foreground and background values are grouped in the presentation of results. Selecting one of the elements in the list highlights it in the page.

\section{THE STUDY}

In order to test the reliability and the limitations of the selected tools we created a test suite. The tests were designed to evaluate from the most simple usage of styles in web pages, to edge cases that might be challenging for automated tools to evaluate. Some of the tests in the suite were directly inspired by the test cases in the ACT Rule "Text has minimum contrast"7. Further work from the ACT Rules Community Group could lead to an updated test suite. In this section we first introduce our test suite before presenting the results of the tests.

\footnotetext{
${ }^{5}$ https://chrome.google.com/webstore/detail/wcag-color-contrastcheck/plnahcmalebffmaghcpcmpaciebdhgdf?hl=en

${ }^{6}$ https://addons.mozilla.org/en-US/firefox/addon/wcag-contrast-checker/

${ }^{7}$ https://act-rules.github.io/rules/afw $4 \mathrm{f} 7$
} 


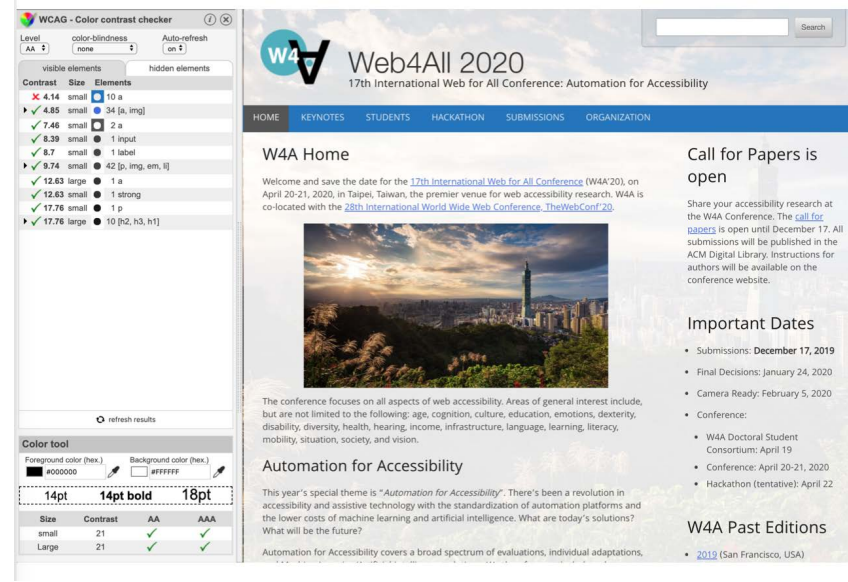

Figure 3: Screenshot of the evaluation of the W4A home page with the WCAG Color Constrast Checker.

\subsection{Test Criteria}

For each test in our test suite, we determined if the tested element passes or fails WCAG success criterion 1.4.3 Contrast (Minimum). To judge conformity to the success criterion we determined the lowest possible contrast between the color of all pixels in the text of the element and the color of all pixels immediately adjacent to the text of the element. If for any pixel in the text the lowest contrast is bellow the WCAG recommendation, the test fails. The formula for calculating the contrast between text and background can be found in the text of Technique G18: Ensuring that a contrast ratio of at least 4.5:1 exists between text (and images of text) and background behind the text.

\subsection{Test Suite}

The test suite divides the tests into 6 categories by varying the styles of the text or of the background: 1) text with a solid color on a background with solid color; 2) text with a solid color on a gradient background; 3) text on an opaque background or text with shadow; 4) text with images as background; 5) text within buttons; and 6) text within different components used in web pages (breadcrumbs, collapsed panels, dropdowns, forms and tables).

Due to space limitations, it is not possible to describe here in detail all tests. However, the reader can access the entire test suite at https://www.di.fc.ul.pt/ cad/contrast_checks/.

\subsection{Results}

All tests were executed on Google Chrome Version 73.0.3683.75 (Official Build) (64-bit) opened on a 1920x1080 window.

The following six tables present the results of the tests in each group. Each table presents the expected contrast according to our criteria and the contrast returned by each of tested tools. Some of the tools do not return a contrast value when they do not flag a WCAG violation or when they are unable to compute the contrast. In those instances we represent the result as not available (N/A).
Table 1: Text with a solid color on a background with solid color

\begin{tabular}{|c|c|c|c|c|}
\hline & Expected & CCAV & WAVE & CCC \\
\hline$\# 1.1$ & 21 & 21 & N/A & 21 \\
\hline$\# 1.2$ & 21 & N/A & N/A & 21 \\
\hline$\# 1.3$ & 21 & N/A & N/A & 21 \\
\hline$\# 1.4$ & 3.98 & 3.97 & 3.97 & 3.98 \\
\hline$\# 1.5$ & 1.16 & 1.16 & 1.16 & 1.16 \\
\hline$\# 1.6$ & 1.26 & 1.25 & 1.25 & 1.26 \\
\hline$\# 1.7$ & 3.72 & 3.72 & 3.72 & 3.73 \\
\hline
\end{tabular}

Table 2: Text with a solid color on a gradient background

\begin{tabular}{|c|c|c|c|c|}
\hline & Expected & CCAV & WAVE & CCC \\
\hline$\# 2.1$ & 3.36 & 21 & N/A & 21 \\
\hline$\# 2.2$ & 1.33 & 21 & N/A & 21 \\
\hline$\# 2.3$ & 2.8 & N/A & N/A & 21 \\
\hline$\# 2.4$ & 1.4 & 1 & N/A & 1 \\
\hline$\# 2.5$ & 1.11 & 2.5 & N/A & 21 \\
\hline
\end{tabular}

Table 3: Text on an opaque background or text with shadow

\begin{tabular}{|c|c|c|c|c|}
\hline & Expected & CCAV & WAVE & CCC \\
\hline$\# 3.1$ & 8.94 & 4.42 & 4.42 & 4.43 \\
\hline$\# 3.2$ & 3.28 & N/A & 4.42 & 4.43 \\
\hline$\# 3.3$ & 21 & 21 & N/A & 21 \\
\hline$\# 3.4$ & 11.42 & 11.42 & N/A & 11.42 \\
\hline$\# 3.5$ & 5.42 & 5.31 & N/A & 5.24 \\
\hline$\# 3.6$ & 2.03 & 2.02 & N/A & 1.99 \\
\hline$\# 3.7$ & 1 & 1 & 1 & 1 \\
\hline
\end{tabular}

Table 4: Text with images as background

\begin{tabular}{|c|c|c|c|c|}
\hline & Expected & CCAV & WAVE & CCC \\
\hline$\# 4.1$ & 11.78 & 1 & 1 & 1 \\
\hline$\# 4.2$ & 1.66 & 1.22 & 1 & 1 \\
\hline$\# 4.3$ & 21 & 21 & N/A & 21 \\
\hline$\# 4.4$ & 1 & N/A & 1 & 1 \\
\hline$\# 4.5$ & 1.5 & 1.22 & 1.22 & 1.23 \\
\hline$\# 4.6$ & 2.12 & N/A & 1 & 1 \\
\hline$\# 4.7$ & 2.8 & 9.41 & N/A & 9.42 \\
\hline$\# 4.8$ & 1 & 1 & 1 & 1 \\
\hline
\end{tabular}

\section{DISCUSSION}

All tools tested excelled in the tests with a well-defined background and text color (table 1). The minor differences found in some tests (one hundredth differences in computed contrast ratios) might be due to rounding errors in the contrast ratio computation process.

However, we found there are clear limitations when the background is no longer solid. The first situation tested considering "dynamic" background colors corresponded to applying a lineargradient to the background CSS property of the element with the solid text. In this instance (table 2), the tools did not detect the 
Table 5: Text within buttons

\begin{tabular}{|c|c|c|c|c|}
\hline & Expected & CCAV & WAVE & CCC \\
\hline$\# 5.1$ & 3.98 & 3.97 & 3.97 & 3.98 \\
\hline$\# 5.2$ & 4.53 & 4.52 & N/A & 4.53 \\
\hline$\# 5.3$ & 1.05 & 1.05 & 1.05 & 1.05 \\
\hline$\# 5.4$ & 11.51 & 11.5 & N/A & 11.51 \\
\hline$\# 5.5$ & 3.98 & 3.97 & 3.97 & 3.98 \\
\hline$\# 5.6$ & 4.53 & 4.52 & N/A & 4.53 \\
\hline$\# 5.7$ & 19.92 & 14.63 & N/A & 14.63 \\
\hline$\# 5.8$ & 19.92 & 11.5 & N/A & 11.51 \\
\hline$\# 5.9$ & 2.43 & N/A & 3.97 & 3.98 \\
\hline$\# 5.10$ & 2.46 & 4.68 & N/A & 4.69 \\
\hline
\end{tabular}

Table 6: Text within different web page components

\begin{tabular}{|c|c|c|c|c|}
\hline & Expected & CCAV & WAVE & CCC \\
\hline$\# 6.1$ & 3.32 & 3.35 & 3.35 & 3.36 \\
\hline$\# 6.2$ & 3.95 & 3.95 & 3.95 & 3.95 \\
\hline$\# 6.3$ & 7.9 & 9.46 & N/A & 9.46 \\
\hline$\# 6.4$ & 21 & N/A & N/A & 15.43 \\
\hline$\# 6.5$ & 3.1 & 3.13 & 3.13 & 3.13 \\
\hline$\# 6.6$ & 4.06 & N/A & N/A & 4.53 \\
\hline$\# 6.7$ & 11.63 & N/A & N/A & N/A \\
\hline$\# 6.8$ & 1 & N/A & N/A & N/A \\
\hline$\# 6.9$ & 11.52 & 11.5 & N/A & 11.51 \\
\hline
\end{tabular}

gradient as the background color of the text, so they compared the foreground color with a solid color for the background.

The following group of tests (table 3) considered first text with the text-shadow CSS property applied with different colors. This was followed by a group of tests with different levels of opacity for the background color. In what concerns the component's opacity control, the tools performed well, taking the opacity level into account in the computation of the color contrast. However, the same tools do not seem to take into account the text shadow. Their results are similar for shadows of different colors. In particular, they all failed the white shadow test that makes text readable while they flag it a WCAG violation.

It was already known beforehand that the tools could not detect images as background (or that they do not use them to calculate the contrast with overlaid text). Consequently, it was no surprise that the computed contrast ratios (table 4) in those instances where the image had a different color from the background color of its parent element were different from the expected contrast ratios. One easy way to confirm this situation is that all values of computed contrast ratios by the different tools when the text was white or black are always one or 21 .

The last two groups of tests (tables 5 and 6) investigated whether the text being the label of a widget impacted the color contrast calculation. In the majority of instances, the tools behaved according to expectations. However, some specific cases raised issues with the tools. For example, components that are hidden (via the CSS property display with value of none) are only flagged by the browser extension (CCC). The two other tools miss these elements, which might become visible as the result of the execution of a script (triggered by an user action or automated means). Another test case that challenged the tools ability refers to placeholder text (inserted in an input field via the placeholder attribute). In this instance all tools were unable to compute the color contrast.

\section{CONCLUSION}

This paper contributed a study of the performance of automated color contrast checking tools. The study confirmed the ability of these tools to correctly check the contrast in most situations, from the simple text with solid color over a solid color background to more complex instances (e.g. background with opacity). The study also identified limitations with the tools. The major limitation relates to the use of images as background. None of the tested tools was able to cope with this situation. The tools performance when a gradient was used in a background was also unsatisfactory. Other styles also led to similar issues (e.g. text-shadow). Finally, the tools' ability to check color contrast for text inside interactive components is not uniform, with some widgets being computed incorrectly.

Another contribution from the paper is the test suite that can be used by developers of contrast checking tools to evaluate the performance of their tools in some edge cases that are currently not adequately addressed.

\section{ACKNOWLEDGMENTS}

This work was supported by the WAI-Tools Project, co-funded by the European Commission (EC) H2020 GA 780057. This work was supported by FCT through funding of the LASIGE Research Unit, ref. UIDB/00408/2020.

\section{REFERENCES}

[1] Ricardo José de Araújo, Julio Cesar Dos Reis, and Rodrigo Bonacin. 2018. Understanding interface recoloring aspects by colorblind people: a user study. Universal Access in the Information Society (14 Aug 2018). https://doi.org/10.1007/s10209018-0631-7

[2] Alessio Facchin, Roberta Daini, and Daniele Zavagno. 2017. The Glare Effect Test and the Impact of Age on Luminosity Thresholds. Frontiers in Psychology 8 (2017), 1132. https://doi.org/10.3389/fpsyg.2017.01132

[3] Fredrik Hansen, Josef Jan Krivan, and Frode Eika Sandnes. 2019. Still Not Readable\&\#63; An Interactive Tool for Recommending Color Pairs with Sufficient Contrast Based on Existing Visual Designs. In The 21st International ACM SIGACCESS Conference on Computers and Accessibility (ASSETS '19). ACM, New York, NY, USA, 636-638. https://doi.org/10.1145/3308561.3354585

[4] Luke Jefferson and Richard Harvey. 2006. Accommodating Color Blind Computer Users. In Proceedings of the 8th International ACM SIGACCESS Conference on Computers and Accessibility (Assets '06). ACM, New York, NY, USA, 40-47. https: //doi.org/10.1145/1168987.1168996

[5] A. Mereuta, S. Aupetit, N. Monmarché, and M. Slimane. 2014. Web Page Textual Color Contrast Compensation for CVD Users Using Optimization Methods. fournal of Mathematical Modelling and Algorithms in Operations Research 13, 4 (01 Dec 2014), 447-470. https://doi.org/10.1007/s10852-013-9239-3

[6] Frode Eika Sandnes and Anqi Zhao. 2015. An Interactive Color Picker that Ensures WCAG2.0 Compliant Color Contrast Levels. Procedia Computer Science 67 (2015), 87 - 94. https://doi.org/10.1016/j.procs.2015.09.252 Proceedings of the 6th International Conference on Software Development and Technologies for Enhancing Accessibility and Fighting Info-exclusion.

[7] Garreth W. Tigwell, David R. Flatla, and Neil D. Archibald. 2017. ACE: A Colour Palette Design Tool for Balancing Aesthetics and Accessibility. ACM Trans. Access. Comput. 9, 2, Article 5 (Jan. 2017), 32 pages. https://doi.org/10.1145/3014588

[8] Lauren E. Welbourne, Antony B. Morland, and Alex R. Wade. 2015. Human colour perception changes between seasons. Current Biology 25, 15 (2015), R646 - R647. https://doi.org/10.1016/j.cub.2015.06.030

[9] Anja Zorko, Snježana Ivančić Valenko, Mario Tomiša, Damira Keček, and Darijo Cerepinko. 2017. The impact of the text and background color on the screen reading experience. Tehnički glasnik 11, 3 (2017), 78-82. https://hrcak.srce.hr/186649 